\title{
Communication assessment scale for advanced dementia: Psychometric properties
}

\author{
Ladislav Volicer $^{1 *}$ and Beverley A Manzar ${ }^{2}$ \\ ${ }^{1}$ School of Aging Studies, University of South Florida, USA \\ ${ }^{2}$ Ebury Court Care Home, Romford, Essex, USA
}

\begin{abstract}
Background and purpose: Communication difficulties are some of the most disturbing symptoms of Alzheimer's disease and other progressive degenerative dementias. There is limited evidence that some interventions may improve communication but a scale that would be easy to administer and include non-verbal communication is not available. This paper describes such a scale and its psychometric properties.
\end{abstract}

Participants: Fifty-one residents of Ebury Court Care Home who are regularly monitored for cognitive status characteristics.

Methods: Communication Assessment for Advanced Dementia (CASAD) scale was based on Boston Aphasia Examination scale. Participants were tested by CASAD and their cognitive status was evaluated by the Mini-mental State examination (MMSE).

Results: CASAD scores were found to be strongly correlated to MMSE scores, and can differentiate subjects even with MMSE scores 0. CASAD has excellent internal consistency with Cronbach alpha equal 0.898, moderate interrater reliability with non-significant difference between scores of two independent raters. Factor analysis showed that CASAD has only one factor, explaining $77.9 \%$ of variance, on which all four items load very highly. CASAD scores are highly reproducible as indicated by repeated testing.

Conclusion: CASAD is easily administered scale, that can be used for evaluation of communication abilities even in persons with most severe dementia.

\section{Introduction}

Communication impairment develops during the progression of Alzheimer's disease $(\mathrm{AD})$ and other progressive degenerative dementias in all patients and is one of the most bothersome symptoms. In the early stages, the patient may develop anomic aphasia that is characterized by word-finding difficulties and paraphasias. As the disease progresses, patients acquire transcortical sensory aphasia, in which comprehension is affected in addition to more severe anomia. Patients may also have decreased language output, echolalia and repetition of meaningless words of phrases (verbal stereotypes) [1]. In severe stages of dementias, patients are unable to communicate verbally and may become mute. However, they may still retain ability to communicate non-verbally.

Impaired communication affects patients with dementia throughout the course of the disease. In a mild stage, patients may be upset when they cannot find words and speak as they used to. As the disease progresses, patients may express their needs non-verbally, often by agitated or disruptive behavior that communicates unmet needs [2]. Communication problems are also a major factor in rejection of care by patients, who do not understand why care needs to be provided and do not comprehend staff explanations [3,4]. Rejection of care can be a major problem for caregivers both at home and in institutions because it may progress into combative/aggressive behavior and often results in administration of psychoactive medications [5].

Several scales were developed for measuring language impairment. The most comprehensive is the Boston Diagnostic Aphasia Examination [6] that has three goals: 1 . diagnosis of presence and type of aphasia, 2. measurement of level of performance and 3. assessment of strength and weaknesses of the patient. The examination is very comprehensive and lengthy and even the short form requires one hour of testing. There is also the Boston Assessment of Severe Aphasia instrument that has 61 items and requires $30-40$ minutes for testing [7]. In our clinical work we felt the need for a short assessment that would also include non-verbal communication. Therefore, we developed Communication Assessment Scale for Advanced Dementia (CASAD) that has only 4 items and requires 3-5 minutes of testing [8], based on an early version of the Boston Aphasia Examination [9]. This paper will report psychometric properties of CASAD.

\section{Methods}

Subjects: All residents $(\mathrm{n}=51)$ of a British care home were participants in a quality improvement activity that included following cognitive functions by administration of Mini-mental State Examination (MMSE) [10] and CASAD. Their ages and gender were obtained from their charts.

Instruments: MMSE is a standard scale used widely for evaluation of severity of dementia. CASAD consists of four items measuring: 1. spontaneous speech, 2. verbal comprehension, 3. naming and 4 . repetition (Appendix). Each item contains five options of answers arranged from the most difficult to no response at all. Subjects are

${ }^{*}$ Correspondence to: Ladislav Volicer, School of Aging Studies, University of South Florida, 2337 Dekan Lane, Land O Lakes, FL 34639, USA, E-mail: lvolicer@usf.edu

Received: May 29, 2018; Accepted: June 08, 2018; Published: June 12, 2018 
asked the most difficult question first and if they cannot answer they are asked the next one. The resulting score is a number of the first correct answer or evaluation of the spontaneous speech and range from 4 (normal communication) to 20 (unable to communicate).

Statistical evaluation: Relationship between MMSE and CASAD was evaluated by the Pearson correlation and by comparing the percentage of subjects who had a measurable score on either scale. Internal consistency of CASAD was determined by calculation of the Cronbach alpha value, interrater reliability by calculation of Cohen's kappa coefficient, and structure of the scale by a factor analysis.

\section{Results}

Subjects included in the study were mostly females (90\%) and their age was $87.5 \pm 5.5$ (mean \pm SD). Fifteen of them had diagnosis of Alzheimer's disease (MMSE scores $0-19$, mean $8.0 \pm 8.5$ ), 22 had diagnosis of vascular dementia (MMSE scores $0-19$, mean $10.3 \pm 6.3$ ) and 14 did not have any diagnosis related to cognitive impairment. Six of those might have been cognitively intact or have only mild dementia (MMSE scores 19 - 30, mean 24.2 \pm 4.5 , CASAD scores $4-5$, mean 4.5 \pm 0.5 ) and 8 were cognitively impaired (MMSE scores $0-11$, mean 5.4 \pm 3.9 , CASAD scores $8-17$, mean $10.4 \pm 3.8$ ). Language impairment was similar in subjects diagnosed with Alzheimer's disease (CASAD scores $4-18$, mean $8.5 \pm 4.0)$ and in subjects diagnosed with vascular dementia (CASAD scores $4-18$, mean $8.0 \pm 4.3$ ).

Figure 1 shows relationship between MMSE and CASAD scores. It shows that MMSE has a bottom effect with 6 participants scoring 0 , and only 1 participant scoring 30 . CASAD has a bottom effect with the smallest score indicating intact communication in 8 subjects with MMSE scores from 16 to 30 but does not have a ceiling effect because all subjects received a score below 20 . There were significant correlations between MMSE and CASAD scores for all subjects $(\mathrm{r}=-0.773, \mathrm{p}<$ 0.001 ) and for the two diagnoses separately (Alzheimer's disease $r=$ $-0.811, \mathrm{p}<0.001$, vascular dementia $\mathrm{r}=-0.806, \mathrm{p}<0.001)$.

Internal consistency analysis showed the CASAD is highly reliable with Cronbach alpha equal 0.898 , with moderate interrater reliability (kappa coefficient $=0.538, \mathrm{p}=0.001$ ), and non-significant difference between scores of two independent raters $(7.1$ vs. $6.6, t=1.08, \mathrm{p}=$

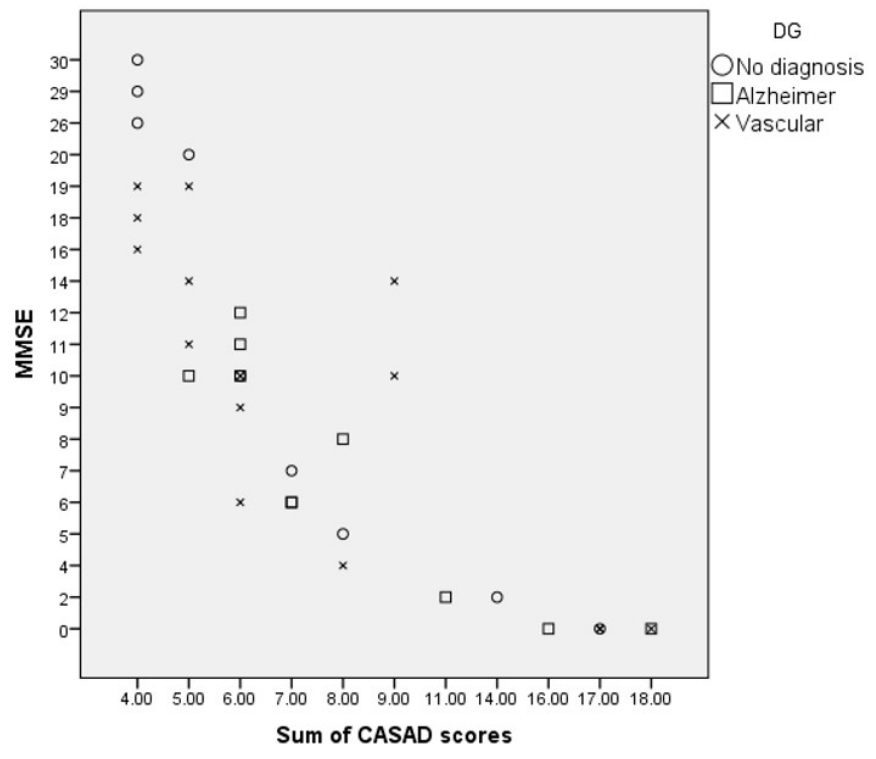

Figure 1. Relationship between MMSE and CASAD scores
0.316). Factor analysis showed that CASAD has only one factor, explaining $77.9 \%$ of variance, on which all four items load very highly (0.83 - 0.94). Reproducibility of CASAD was tested by comparing mean CASAD scores obtained two months apart in residents whose MMSE score did not change. The mean scores were identical (7.09) and were highly correlated $(\mathrm{r}=0.913, \mathrm{p}=004)$. In a past research, CASAD was shown to be sensitive in detecting deterioration of language caused by seizures [11].

\section{Discussion}

The importance of communication difficulties in dementia is recognized by many investigators. Several studies tried to improve communication by educating care providers in communication strategies. These strategies included elimination of elderspeak [12], use of memory books [13] and specialized programs [14,15]. Although these strategies were successful in improving behavior of nursing home staff, these studies did not measure a possible effect of these strategies on communication abilities of persons with dementia. This implied that the investigators did not believe that the communication abilities of persons with dementia could improve, despite the fact that a tele rehabilitation program that involved individuals with very early stage Alzheimer's disease documented improvement in language abilities [16].

Another reason for omitting evaluation of effects of these programs on persons with dementia could have been the lack of an easy to use scale for measuring communication abilities of persons with advanced dementia. As explained in the Introduction, currently available scales are very time consuming and may not be applicable to persons with advanced dementia. CASAD eliminates these shortcomings and can be used readily in programs that try to improve quality of life of persons with dementia. An example of such a program is Namaste Care, where interviews with the staff and family members indicated significant improvement of communication abilities [17]. It would be important to support these subjective evaluations by quantitative data.

Because of the ceiling effect, CASAD is not useful in studies that include persons with a mild degree of dementia. However, CASAD score may be calculated in almost all persons with advanced dementia. In the current study, 6 participants with MMSE score of 0 had CASAD score ranging from 2 to 4 . Another study also showed that CASAD score could be calculated in a majority of persons with MMSE score of 0 [18]. Measurement of communication ability is important in evaluating the effects of programs designed to the improve quality of life of people with advanced dementia.

This study has several limitations. We did not have diagnoses for some of the subjects with clear cognitive impairment. However, the scale performed equally well with subjects with diagnosis of Alzheimer's disease and diagnosis of vascular dementia. We did not test reproducibility of CASAD scores and this will require further research. However, we would like to make research community aware of CASAD and make it available because of increasing interest in studies that include persons with advanced dementia (Appendix).

\section{References}

1. Feldman HH, Woodward M (2005) The staging and assessment of moderate to severe Alzheimer disease. Neurology 65: S10-S17.

2. Algase DL, Beck C, Kolanowski A, Whall A, Berent S, et al. (1996) Need-driven dementia-compromised behavior: an alternative view of disruptive behavior. $\mathrm{Am} \mathrm{J}$ Alzheim Dis 11: 10-19.

3. Galindo-Garre F, Volicer L, Van der Steen JT (2015) Factors related to rejection of care and behaviors directed towards others: a longitudinal study in nursing home residents with dementia. Dement Geriatr Cogn Dis Extra 5: 123-134. [Crossref] 
4. Volicer L, Van der Steen JT, Frijters DH (2009) Modifiable factors related to abusive behaviors in nursing home residents with dementia. J Am Med Dir Assoc 10: 617-622. [Crossref]

5. Ishii S, Streim JE, Saliba D (2012) A conceptual framework for rejection of care behaviors: review of literature and analysis of role of dementia severity. J Am Med Dir Assoc. 11-23. [Crossref]

6. Goodglass H, Kaplan E, Barresi B (2001) Boston Diagnostic Aphasia Examination. 3rd: Philadelphia, PA: Lippincott, Williams \& Wilkins.

7. Helm-Estabrooks N, Ramsberger G, Morgan AR, Nicholas M (2015) Boston Assessment of Severe Aphasia. p. 1625

8. Volicer L, Seltzer B, Rheaume Y, Fabiszewski K, Herz L, et al. (1987) Progression of Alzheimer-type dementia in institutionalized patients: a cross-sectional study. J Appl Gerontol 6: 83-94.

9. Goodglass H, Kaplan EF (1972) The Assessment of Aphasia and Related Disorders. Lea \& Febiger, Philadelphia.

10. Folstein M, Folstein S, McHugh PJ (1975) 'Mini-mental State,' a practical method for grading the cognitive state of patients for clinicians. J Psychiatr Res 12: 189-198. [Crossref]
11. Volicer L, Smith S, Volicer BJ (1995) Effect of seizures on progression of dementia of the Alzheimer type. Dementia 6: 258-263. [Crossref]

12. Williams KN (2006) Improving outcomes of nursing home interactions. Res Nurs Health 29: 121-133. [Crossref]

13. Bourgeois MS, Dijkstra K, Burgio LD, Allen RS (2004) Communication skills training for nursing aides of residents with dementia. Clin Gerontol 27: 119-138.

14. McCallion P, Toseland RW, Lacey D, Banks S (1999) Educating nursing assistants to communicate more effectively with nursing home residents with dementia. Gerontologist 39: 546-558. [Crossref]

15. Ripich DN, Wykle M, Niles S (1995) Alzheimer's disease caregivers: the focused program. A communication skills training program helps nursing assistants to give better care to patients with disease. Geriatr Nurs 16: 15-19.

16. Jelcic N, Agostini M, Meneghello F, Busse C, Parise S, et al. (2014) Feasibility and efficacy of cognitive telerehabilition in early Alzheimer's disease: a pilot study. Clinical Interventions in Aging 9: 1605-1611.

17. Manzar BA, Volicer L (2015) Increased quality of life in advanced dementia by Namaste Care: grounded theory analysis. Am J Alzheim Dis 2: 24-37.

18. Volicer L, Hurley AC, Lathi DC, Kowall NW (1994) Measurement of severity in advanced Alzheimer's disease. J Gerontol 49: M223-226. [Crossref]

Copyright: $\odot 2018$ Volicer L. This is an open-access article distributed under the terms of the Creative Commons Attribution License, which permits unrestricted use, distribution, and reproduction in any medium, provided the original author and source are credited. 